\title{
Spin current in the Kondo lattice model
}

\author{
Shun-Qing Shen ${ }^{1}$ and X. C. Xie ${ }^{2}$ \\ ${ }^{1}$ Department of Physics, The University of Hong Kong, Pokfulam Road, Hong Kong, China \\ ${ }^{2}$ Department of Physics, Oklahoma State University, Stillwater, Oklahoma 74078 \\ and International Center for Quantum Structure, Chinese Academy of Sciences, Beijing, China \\ (Received 6 January 2003; revised manuscript received 18 February 2003; published 28 April 2003)
}

\begin{abstract}
By using the projection operator technique it is observed that the strong Hund's rule coupling and $s-d$ interaction in transition metal elements may lead to an effective coupling between the spin current and spin chirality. As a result, the spin chirality can be regarded as a driving force to produce a spin current. The spin current may give rise to a novel type of field acting on the spins. A spin battery is designed based on the interactions between the spin current and spin chirality.
\end{abstract}

DOI: 10.1103/PhysRevB.67.144423

PACS number(s): 75.10.Lp, 72.15.Gd

Spin-dependent effects, such as giant or colossal magnetoresistance and magnetization switching, arise from the interaction between the spin of the charge carrier and an applied external magnetic field. In spintronics, instead of charge, electron spin carries information and it is possible that capability and performance can be enhanced in spintronics devices. ${ }^{1-3}$ Possible applications and the fundamental science involved make the study of spin-dependent transport an intensive field in condensed matter physics. Recently, several ways to produce a pure spin current were proposed, such as the anomalous Hall effect in ferromagnetic metals, ${ }^{4,5}$ ferromagnetic resonance, ${ }^{6,7}$ and the spin current in a spin spiral state. $^{8,9}$ In this paper, we study spin current in the Kondo lattice model, a simplified model containing essential physics for many ferromagnetic metals or semiconductors. We find a type of interaction between the spin current and spin chirality (defined later) in the strong Hund coupling limit. This indicates that the spin chirality can be regarded as a driving force to produce spin current, similar to what an electric field does to the electric current. Furthermore, the spin current induces a type of effective magnetic field. Based on the interaction between spin current and spin chirality, we propose a scheme to design a spin battery. As a demonstration, we study the spin transport in the well known spiral state to bring out the above mentioned spin-current effects.

The Hund's rule coupling and $s$ - $d$ exchange interaction are crucial in transition metal elements. They play a key role in the formation of metallic ferromagnetism in colossal magnetoresistance materials ${ }^{10-12}$ and diluted magnetic semiconductors. $^{13,14}$ In this paper we focus on doped transition metal oxides and/or ferromagnetic semiconductors. The minimal model for these materials is the Kondo lattice model,

$$
H=-t \sum_{\langle n, m\rangle, \sigma} c_{n, \sigma}^{\dagger} c_{m, \sigma}-\frac{J}{2} \sum_{n, \sigma \sigma^{\prime}} \mathbf{S}_{n} \cdot \tau_{\sigma \sigma^{\prime}} c_{n, \sigma}^{\dagger} c_{n, \sigma^{\prime}},
$$

where $c_{n, \sigma}^{\dagger}$ and $c_{n, \sigma}$ are the creation and annihilation operator for conduction electrons, $\mathbf{S}_{n}$ is the spin operators for the localized spin, $\tau$ is the Pauli matrix, and $\tau_{0}$ is the $2 \times 2$ identity matrix. The summation over $\langle n, m\rangle$ runs for the nearest neighbor pairs of lattice sites. In this paper we will set $\hbar=c=1$, and the lattice space is $a$ and is set to unit. ${ }^{15}$
For the Hund's rule coupling, $J$ is always positive. The model has been studied extensively. ${ }^{16}$ The spin current operator along the $\alpha$ axis in the tight binding approximation is defined as $t \hbar \mathbf{I}_{n n+\alpha}^{s}$, and

$$
\mathbf{I}_{n n+\alpha}^{s}=-\frac{i}{2} \sum_{\sigma, \sigma^{\prime}}\left(c_{n, \sigma^{\prime}}^{\dagger} \tau_{\sigma \sigma^{\prime}} c_{n+\alpha, \sigma^{\prime}}-c_{n+\alpha, \sigma^{\dagger}}^{\dagger} \tau_{\sigma \sigma^{\prime}} c_{n, \sigma^{\prime}}\right),
$$

where $\alpha(=x, y, z)$ are vectors pointing to the nearest neighbor sites, which have one longitudinal component and two transverse components. The spin polarized currents and electric current become equal in a fully polarized ferromagnetic system.

Before we discuss the spin transport of Eq. (1) in an arbitrary dimension, let us first illustrate the physics by considering the well known spin spiral state in a onedimensional system. In general, the spin spiral state may not be the ground state of Eq. (1); however, it can be stabilized by external environments, such as by applying external fields along different directions at the ends of the system. ${ }^{17}$ Our purpose here is to demonstrate the consequence of the spin spiral state. We parametrize $\mathbf{S}_{n}$ by the polar angles $\theta_{n}$ and $\varphi_{n}$, i.e., making the classical spin approximation. The spin spiral state is defined by $\mathbf{S}_{n} / S$ $=(\sin \theta \cos n \varphi, \sin \theta \sin n \varphi, \cos \theta)$. The spin chirality defined as the cross times between the two neighboring spins: $\left\langle\mathbf{S}_{n}\right.$ $\left.\times \mathbf{S}_{n+1}\right\rangle_{z}=S^{2} \sin ^{2} \theta \sin \varphi$. In the $k$ space, we introduce a spinor operator $\Phi^{\dagger}(k)=\left(c_{k a+\varphi / 2, \uparrow}^{\dagger}, c_{k a-\varphi / 2, \downarrow}^{\dagger}\right)$. The mean field Hamiltonian is written as

$$
H=\sum_{k} \Phi^{\dagger}(k) H(k) \Phi(k)
$$

where $\quad H(k)=-g_{0} \tau_{0}-g_{x} \tau_{x}-g_{z} \tau_{z} \quad$ with $\quad g_{0}=\mu$ $+2 t \cos (\varphi / 2) \cos k a, \quad g_{x}=(J / 2) \sin \theta$, and $g_{z}=(J / 2) \cos \theta$ $-2 t \sin (\varphi / 2) \sin k a$. $\mu$ is the chemical potential. The single particle Green function in a $2 \times 2$ matrix form is given by

$$
\begin{aligned}
G\left(k, i \omega_{n}\right) & =\left[i \omega_{n} \tau_{0}-H(k)\right]^{-1} \\
& =\frac{\left[\left(i \omega_{n}+g_{0}\right) \tau_{0}-g_{x} \tau_{x}-g_{z} \tau_{z}\right]}{\left(i \omega_{n}-\omega_{+}+\mu\right)\left(i \omega_{n}-\omega_{-}+\mu\right)},
\end{aligned}
$$




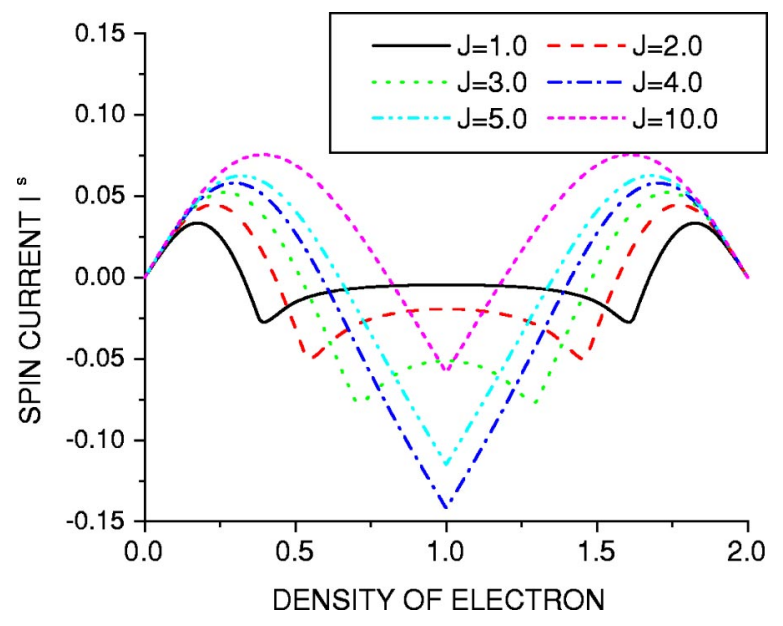

FIG. 1. Spin current via the density of the conduction electron in the spin spiral state of $\theta=\pi / 2$ and $\varphi=0.2 \pi$.

where $\omega_{n}=(2 n+1) \pi k T$ with an integer $n$ and $T$ is the temperature. The two branches of spectra are

$$
\begin{aligned}
\omega_{ \pm}(k)= & -2 t \cos \frac{\varphi}{2} \cos k a \pm\left[\left(\frac{J}{2} \cos \theta-2 t \sin \frac{\varphi}{2} \sin k a\right)^{2}\right. \\
& \left.+\frac{J^{2}}{4} \sin ^{2} \theta\right]^{1 / 2} .
\end{aligned}
$$

In the $k$ space, the expectation value of the longitudinal spin current operator is written in terms of the Green's function,

$$
\left\langle I_{z}^{S}\right\rangle=\frac{1}{N} \sum_{k}\left[A_{+} n_{F}\left(\omega_{+}-\mu\right)-A_{-} n_{F}\left(\omega_{-}-\mu\right)\right],
$$

where

$$
\begin{aligned}
A_{ \pm}(k)= & \pm \sin \frac{\varphi}{2} \cos k a \\
& +\frac{t \sin \varphi \sin ^{2} k a-\frac{J}{2} \cos \theta \cos \frac{\varphi}{2} \sin k a}{\sqrt{\left(\frac{J}{2} \cos \theta-2 t \sin \frac{\varphi}{2} \sin k a\right)^{2}+\frac{J^{2}}{4} \sin ^{2} \theta}} .
\end{aligned}
$$

We also calculated the electric current $I_{z}^{c}$ and found that $I_{z}^{c}$ $=0$. Thus in the spiral state there exists a spin current without a charge current. In Fig. 1 we plot the spin current as a function of the electron density in the state with a $\theta=\pi / 2$, and $\varphi=0.2 \pi$ for different $J$. We see that a larger $J$ produces a higher spin current. For a finite $J$ the current may change direction with change of density. In Fig. 2 we plot the relation between the spin current and spin chirality at the density of 0.2. The spin current increases with the spin chirality.

In the large $J$ limit, the problem can be simplified. An equivalent Hamiltonian of Eq. (1) or so-called double exchange model in the large $J$ limit is given by ${ }^{18}$

$$
H_{d e}=-\sum_{\langle n, m\rangle} t_{n m} \alpha_{n}^{+} \alpha_{m}
$$

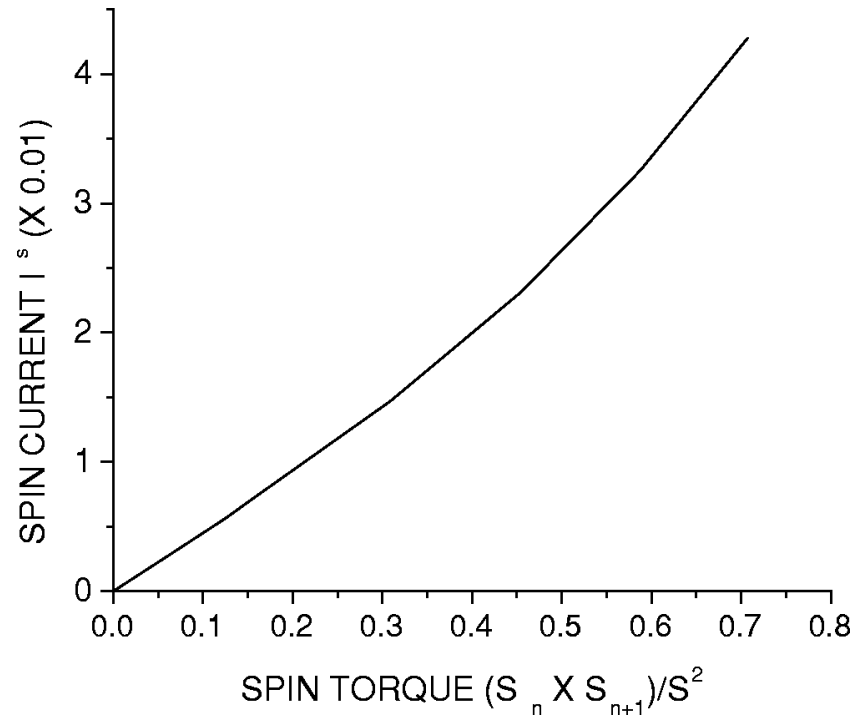

FIG. 2. Spin current via spin spirality at $J=1.0$ with the density of conduction electron 0.20 .

with

$$
t_{n m}=t\left[\cos \frac{\theta_{n}}{2} \cos \frac{\theta_{m}}{2}+\sin \frac{\theta_{n}}{2} \sin \frac{\theta_{m}}{2} e^{-i\left(\varphi_{n}-\varphi_{m}\right)}\right],
$$

and $\quad \alpha_{n}=\cos \left(\theta_{n} / 2\right)\left(1-n_{n \downarrow}\right) c_{n \uparrow}+\exp \left[i \varphi_{n}\right] \sin \left(\theta_{n} / 2\right)(1$ $\left.-n_{n \uparrow}\right) c_{n \downarrow}$ and satisfies the anticommutation relation, $\left[\alpha_{k}, \alpha_{k^{\prime}}^{\dagger}\right]=\delta_{k, k^{\prime}}$. The energy excitations and their eigenstates can be obtained from the diagonalized Hamiltonian

$$
H_{d e}=-2 t \sum\left[\cos ^{2} \frac{\theta}{2} \cos k a+\sin ^{2} \frac{\theta}{2} \cos (k a+\varphi)\right] \alpha_{k}^{\dagger} \alpha_{k},
$$

where $\alpha_{k}$ is the Fourier transform of $\alpha_{n}$. In the case when the spins of conduction electrons are frozen by the localized spins, and the quasiparticles become spinless fermions. The two Fermi momenta are $k_{ \pm}^{F} a=-k_{0} \pm \rho \pi$ where $k_{0}$ $=\arctan \left[\sin ^{2} \theta \sin \varphi /\left(\cos ^{2} \theta+\sin ^{2} \theta \cos \varphi\right)\right]$, and $\rho$ is the ratio of the number of conduction electrons to the number of total lattice sites and $\rho / a$ is the density of conduction electrons. $k_{0}$ is nonzero when the order parameter for the spin spiralling is nonzero. Its lowest energy state is $|\mathrm{GS}\rangle=\prod_{k_{F}^{-} \leqslant k \leqslant k_{F}^{+}} \alpha_{k}^{\dagger}|0\rangle$. The spin current in the state can be calculated from $\mathbf{I}_{z}^{s}$ $=\Sigma_{n}\left\langle\mathrm{GS}\left|\mathbf{I}_{n n+1}^{s}\right| \mathrm{GS}\right\rangle / N$. We have an analytical expression for the spin current:

$$
I_{z}^{s}=t \hbar \frac{\sin \rho \pi}{\pi} \frac{\sin ^{2} \theta \sin \varphi}{\sqrt{1-\sin ^{2} \theta \sin ^{2}(\varphi / 2)}} .
$$

The result is equal to what we obtain by taking the large limit in Eq. (6). In the point of view of the Berry phase the spin spiral state acquires a constant Berry phase in the spindependent renormalized factor in the Hamiltonian [Eq. (7)], i.e., $t_{n n+1}=\left|t_{n n+1}\right| e^{-i \delta \varphi}$. The nonzero phase $\delta \varphi$ drives a spin current.

By analogy with electric field and resistance, we introduce the concept of spin current resistance or its reciprocal, 
spin conductance. The role of $\mathbf{S}_{n} \times \mathbf{S}_{m}$ here is analogous to the local electric field. In the spin spiral state the spin chirality is nonzero, $\left\langle\mathbf{S}_{n} \times \mathbf{S}_{n+1}\right\rangle_{z}=S^{2} \sin ^{2} \theta \sin \varphi$. In Eq. (9) the current is proportional to the spin chirality $\left\langle\mathbf{S}_{n} \times \mathbf{S}_{n+1}\right\rangle_{z}$, and can be written in a compact form $I_{z}^{s}=c \cdot\left\langle\mathbf{S}_{n} \times \mathbf{S}_{n+1}\right\rangle_{z} / S^{2}$ by introducing a spin conductance

$$
c=\sin \rho \pi /\left[\pi \sqrt{1-\sin ^{2} \theta \sin ^{2}(\varphi / 2)}\right]
$$

in a large $J$ limit. The spin conductance is determined by the filling of electrons and the magnetic structure. Just like electric conductance, the spin conductance reflects intrinsic properties of spin transport in materials. It depends on the spindependent scattering mechanism of electrons. In Eq. (10), for the case of $\rho \rightarrow 0$ or $\rightarrow 1, c$ is proportional to the density of electrons $\rho$ or the density of holes $1-\rho$. For a small spin chirality $\sin ^{2} \theta \sin ^{2}(\varphi / 2) \rightarrow 0$, the spin conductance approaches $[\sin \rho \pi] / \pi$, which is proportional to the averaging kinetical energy of the charge carriers in this tight binding theory. From the numerical results in Figs. 1 and 2, we deduce that the spin conductance also depends on $J$. It becomes more complicated for the finite $J$ cases. Similarly, we can also introduce a spin voltage, which is also proportional to the length along the spin spiraling path, $V_{\text {spin }}=\int\left\langle\mathbf{S}_{n}\right.$ $\left.\times \mathbf{S}_{n+1}\right\rangle \cdot d \mathbf{l} / S^{2}$ where $d \mathbf{l}=\mathbf{r}_{n+1}-\mathbf{r}_{n}$ is the difference of two position vectors of neighboring spins. $V_{\text {spin }}$ is an important parameter characterizing the ability to produce a spin current.

Thus far we have introduced several important concepts, such as spin chirality or spin current resistance, through the example of the classical spiral state. To determine the microscopic mechanism to produce a spin current, we go back to the Kondo lattice model [Eq. (1)] in the large Hund's rule coupling limit, and address the quantum spin case. The following discussions are not limited to one-dimensional systems and the conclusion is independent of the dimensionality. According to the Hund's rule coupling, the energy of empty or double occupancy of conduction electrons is zero. For a single occupancy the conduction electron and localized spin can form a spin $S+1 / 2$ state with $-J S / 2$ and a spin $S$ $-1 / 2$ state with $+J(S+1) / 2$. In the large $J$ limit the spin $S-1 / 2$ state and double occupancy should be excluded as they have much higher energy than the spin $S+1 / 2$ state. This process can be realized with the help of the projection technique ${ }^{19}$ For that, we introduce the spinor $\phi_{n}^{+}=[(1$ $\left.\left.-n_{n, \downarrow}\right) c_{n, \uparrow}^{\dagger},\left(1-n_{n, \uparrow}\right) c_{n, \downarrow}^{\dagger}\right]$. The dressed operators (1 $\left.-n_{n,-\sigma}\right) c_{n, \sigma}^{\dagger}$, instead of $c_{n, \sigma}^{\dagger}$, are used here to avoid the double occupancy due to the strong coupling. The projection operator for the spin $S+1 / 2$ state is defined as

$$
\mathcal{P}_{n}^{+}=\phi_{n}^{+} \mathbf{P}_{n}^{+} \phi_{n}
$$

where $\mathbf{P}_{n}^{+}=\left[\mathbf{S}_{n} \cdot \tau+(S+1) \tau_{0}\right] /(2 S+1)$. The operator $\mathcal{P}_{n}^{+}$ forces the electron spin at site $n$ to be fully polarized with the localized spin at the same site. The projection operator for nonoccupancy is $\mathcal{P}_{n}^{e}=\left(1-n_{n, \downarrow}\right)\left(1-n_{n, \uparrow}\right)$. The total projection operator to exclude the spin S-1/2 single occupancy and double occupancy is $\mathcal{P}=\Pi_{n}\left(\mathcal{P}_{n}^{e}+\mathcal{P}_{n}^{+}\right)$. Therefore the equivalent Hamiltonian in the strong Hund's rule coupling limit is expressed as ${ }^{12,20}$

$$
H_{e f f}=\mathcal{P} H \mathcal{P}=-t \sum_{n m}\left[\phi_{n}^{+} \mathbf{P}_{n}^{+} \mathbf{P}_{m}^{+} \phi_{m}+\text { H.c. }\right],
$$

where a constant is omitted in the last step. In the large $S$ approximation the Hamiltonian of [Eq. (12)] becomes the double exchange model in Eq. (7). Using a mathematical identity

$$
\left(\mathbf{S}_{n} \cdot \tau\right)\left(\mathbf{S}_{m} \cdot \tau\right)=\mathbf{S}_{n} \cdot \mathbf{S}_{m} \tau_{0}+i\left(\mathbf{S}_{n} \times \mathbf{S}_{m}\right) \cdot \tau,
$$

the Hamiltonian can be rewritten as ${ }^{21}$

$$
\begin{aligned}
H= & -\frac{(S+1)^{2} t}{(2 S+1)^{2}} \sum_{n m} k_{n m}-\frac{t}{(2 S+1)^{2}} \sum_{n m} k_{n m} \mathbf{S}_{n} \cdot \mathbf{S}_{m} \\
& -\frac{(S+1) t}{(2 S+1)^{2}} \sum_{n m}\left(\mathbf{S}_{n}+\mathbf{S}_{m}\right) \cdot \mathbf{M}_{n m} \\
& +\frac{t}{(2 S+1)^{2}} \sum_{n m}\left(\mathbf{S}_{n} \times \mathbf{S}_{m}\right) \cdot \mathbf{I}_{n m}^{s},
\end{aligned}
$$

where $k_{n m}=\phi_{n}^{+} \tau_{0} \phi_{m}+\phi_{m}^{+} \tau_{0} \phi_{n} \quad$ and $\quad \mathbf{M}_{n m}=\phi_{n}^{+} \tau \phi_{m}$ $+\phi_{m}^{+} \tau \phi_{n}$. The first term is the kinetic part for conduction electrons excluding double occupancy, like the $t$ term in the $t-J$ model. The second term contains an effective Heisenberg-type spin-spin exchange interaction. Approximately, the effective coupling is $J_{\text {ferro }}=-t\left\langle k_{n m}\right\rangle /(2 S$ $+1)^{2}$, proportional to the kinetic energy in the sense of the mean field approximation. The kinetic energy $-t\left\langle k_{n m}\right\rangle$ in the tight binding approximation is always negative if the electron filling $\rho<1$, such that the effective coupling is ferromagnetic. This is consistent with the double exchange picture. $^{22,23}$ The third term is the interaction between local spin $\mathbf{S}_{n}$ and the vector $\mathbf{M}_{n m}$. For a paramagnetic state $\left\langle\mathbf{M}_{n m}\right\rangle$ is equal to zero, but in a fully polarized state its value is equal to $\left\langle k_{n m}\right\rangle$, i.e., proportional to the kinetic energy. Among the four terms the most important observation is from the last term: it contains an interaction between the spin current operator $\mathbf{I}_{n m}^{s}$ and the spin chirality $\mathbf{S}_{n} \times \mathbf{S}_{m}$. By analogy with the coupling between the electric current and electric field it is straightforward to understand why a nonzero spin chirality induces a pure spin current. Although we cannot derive an analytic expression for a finite $J$, it is expected that the mechanism to produce a spin current remains the same. ${ }^{24}$

An effective field and spin battery. To clarify the physical meaning of the last term, we come to calculate the rate of change of a local spin. From classical mechanics or from the Heisenberg equation of motion the rate of change of angular momentum $\mathbf{S}_{n}$ is equal to the torque $\mu_{n} \times \mathbf{h}_{\text {eff }}^{n}$ which acts on the spin,

$$
d \mathbf{S}_{n} / d t=-g \mu_{B} \mathbf{S}_{n} \times \mathbf{h}_{e f f}^{n},
$$

where the effective field experienced by the spin is 


$$
\begin{aligned}
\mathbf{h}_{e f f}^{n}= & \frac{t}{g \mu_{B}(2 S+1)^{2}} \sum_{\delta}\left[k_{n n+\delta} \mathbf{S}_{n+\delta}+(S+1) \mathbf{M}_{n n+\delta}\right. \\
& \left.+\mathbf{I}_{n n+\delta}^{s} \times \mathbf{S}_{n+\delta}\right] .
\end{aligned}
$$

The first two terms, again, are related to the particle's orbital motions. The third term is the torque between the spin current and the local spin. A type of force or effective field is caused due to the interaction between the spin current and local spin or local magnetization. The force may be responsible for magnetization precession by the spin injection into a magnetic conductor ${ }^{25}$ and the anomalous Hall effect in ferromagnetic materials. ${ }^{4,26}$ Further discussion along this line will be published elsewhere. Our observation will lead to potential applications. For example, by means of the spin spiraling properties we can design a spin battery to produce a pure spin current. The battery contains two parts as shown in Fig. 3. The main part is a spin spiraling material. The spins spiral about the axis which is perpendicular to the interface with a ferromagnetic metal film, such that the spin voltage along the axis is nonzero. The spin chirality in the material will produce the spin accumulation at two terminals. The magnetization of the film is also perpendicular to the interface. The film is a source of spins which will flow out of the battery driven by the spin chirality. The key element in this battery is the spin spiraling materials. Possible realizations of spiral states have been discussed by several authors, ${ }^{27-30}$ and

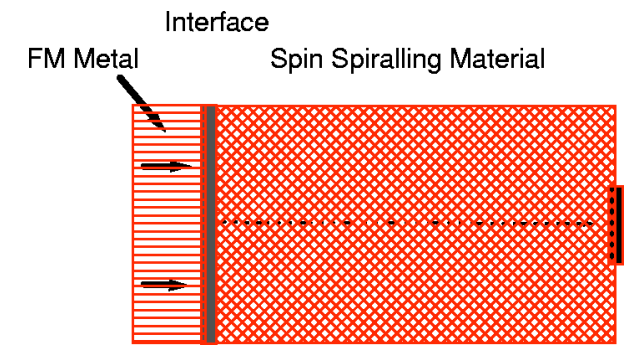

FIG. 3. Schematic view of dc spin battery. Arrows in the ferromagnetic metal as a spin source indicate the magnetization is perperdicular to the interface. The spin spiraling material provides a nonzero spin spirality to drive the spin current along the axis.

the state may also be stabilized with the help of the external magnetic field and exchange coupling between localized spins. In conclusion, we have shown that the strong coupling between the spins of charge carriers and local spins leads to an effective interaction between spin current and spin chirality in the system. A nonzero spin chirality may give rise to a pure spin current, and in return a spin current can cause an interesting type of force on the spins. We confirm these findings by studying in detail the spin transport in the spin spiral state.

The work was supported by grants from the Research Grants Council of Hong Kong, China (Project No. HKU7088/01P) and the U.S. DOE.
${ }^{1}$ A. A. Wolf et al., Science (Washington, DC, U.S.) 294, 1488 (2001).

${ }^{2}$ Spin Electronics, edited by M. Ziese and M. J. Thornton (Springer, New York, 2002).

${ }^{3}$ Semiconductor Spintronics and Quantum Computation, edited by D. D. Awschalom, D. Loss, and N. Samarth (Springer, New York, 2002).

${ }^{4}$ J. E. Hirsch, Phys. Rev. Lett. 83, 1834 (1999); Phys. Rev. B 60, 14787 (1999).

${ }^{5}$ S. Zhang, Phys. Rev. Lett. 85, 393 (2002).

${ }^{6}$ A. Brataas, Y. Tserkovnyak, G. E. W. Bauer, and B. I. Halperin, Phys. Rev. B 66, 060404 (2002).

${ }^{7}$ L. Berger, Phys. Rev. B 59, 11465 (1999); J. Appl. Phys. 90, 4632 (2001).

${ }^{8}$ S. Q. Shen, Phys. Lett. A 235, 403 (1997).

${ }^{9}$ J. Konig, M. C. Bonsager, and A. H. MacDonald, Phys. Rev. Lett. 87, 187202 (2001).

${ }^{10}$ C. Zener, Phys. Rev. 82, 403 (1951).

${ }^{11}$ P. W. Anderson and H. Hasegawa, Phys. Rev. 100, 675 (1955).

${ }^{12}$ K. Kubo and N. Ohata, J. Phys. Soc. Jpn. 33, 21 (1972).

${ }^{13}$ H. Ohno, Science (Washington, DC, U.S.) 281, 951 (1998).

${ }^{14}$ T. Dietl, O. Ohno, F. Matskura, J. Cibert, and D. Ferrand, Science (Washington, DC, U.S.) 287, 1019 (2000).

${ }^{15}$ D. J. Scalapino, S. R. White, and S. Zhang, Phys. Rev. B 47, 7995 (1993).

${ }^{16}$ H. Tsunetsugu, M. Sigrist, and K. Ueda, Rev. Mod. Phys. 69, 809 (1997).
${ }^{17}$ As an illustration we consider a ferromagnetic Heisenberg chain with $n$ spins. The directions of two spins at two terminals can be controled by strong magnetic fields. Assume the angles between the two spins are fixed to be $\varphi$. In the classic spin limit the ground state is obtained by minimizing the classical energy and is shown to be an spin spiral state: the angle between two nearest neighbour spins is $\varphi /(n-1)$. The quantum case can be solved by the Bethe ansatz and/or in the spin wave theory. Other conditions such as longer-range exchange coupling and spin lattice structures may also give arise a spin spiral state.

${ }^{18}$ E. Müller-Hartmann and E. Dagotto, Phys. Rev. B 54, R6819 (1996).

${ }^{19}$ P. Fulde, Electron Correlations in Molecules and Solids (Springer, New York, 1997).

${ }^{20}$ S. Q. Shen and Z. D. Wang, Phys. Rev. B 58, R8877 (1998); 61, 9532 (2000).

${ }^{21}$ The microscopic mechanism should hold in the case of an antiferromagnetic coupling $J<0$ and large S. In the strong limit the conduction electron and local spin form a spin $S-1 / 2$ state. The projection operator for the state is $\mathbf{P}_{n}^{-}=\left[-\mathbf{S}_{n} \cdot \tau+S \tau_{0}\right] /(2 S$ +1 ) instead of $\mathbf{P}_{n}^{+}$. The main different point is that the coupling in the third term in Eq. (14) is antiferromagnetic instead of ferromagnetic, and the correspondent coefficients are revised slightly. The ferromagnetism in diluted magnetic semiconductors originates from the antiferromagnetic coupling between the conduction electrons and doped magnetic elements (Ref. 14).

${ }^{22}$ N. Furukawa, J. Phys. Soc. Jpn. 64, 2734 (1995). 
${ }^{23}$ A. J. Millis, P. B. Littlewood, and B. I. Shraiman, Phys. Rev. Lett. 74, 5144 (1995); H. Röder, J. Zang, and A. R. Bishop, ibid. 76, 1356 (1996).

${ }^{24}$ Apparently the stronger coupling favors to produce the spin current in a spiral state, as shown in Fig. 1.

${ }^{25}$ W. Weber, S. Riesen, and H. C. Siegmann, Science (Washington, DC, U.S.) 291, 1015 (2001).
${ }^{26}$ T. Jungwirth, Qian Niu, and A. H. MacDonald, Phys. Rev. Lett. 88, 207208 (2002).

${ }^{27}$ K. Kubo, J. Phys. Soc. Jpn. 51, 782 (1982).

${ }^{28} \mathrm{~J}$. Jensen and A. R. Machintosh, Rare Earth Magnetism (Clarendon Press, Oxford, 1991).

${ }^{29}$ M. Korling and J. Ergon, Phys. Rev. B 54, 8293 (1996).

${ }^{30}$ J. Inoue and S. Maekawa, Phys. Rev. Lett. 74, 3407 (1995). 